\section{When It's Really Good, We Know It!}

A few weeks ago, an extraordinary announcement came from the FDA. Pembrolizumab, a PD-L1 inhibitor already approved for selected disease indications, received accelerated approval for second-line therapy for any unresectable or metastatic tumor demonstrating microsatellite instability-high (MSI-H) or mismatch DNA repair. This is the first time on record that a drug has been approved for a specific tissue phenotype rather than a specific disease.

The classic condition that shows this phenotype is Lynch syndrome. Years ago, Dr. Henry Lynch of Creighton University observed an unusual clustering of colon cancer, as well as an excess of other cancers, in certain families. This was decades before the molecular underpinnings of the disease were understood. And until now, the primary importance of knowing who carried pathologic germline mutations was for screening and early detection, not disease management.

The success of checkpoint inhibitors (antibodies to CTLA-4, PD-1, or PD-L1) has been transformative in some diseases, generally those with a high mutation burden. Some cancers, however, such as colon and pancreatic cancers, are considered "cold tumors," unresponsive to checkpoint blockade. When I think of how this story unfolded in MSI-H tumors, I almost break into a sweat. Were it not for one unusual patient and some very inquisitive scientists, we might never have gone down this road.

As the story goes, investigators at Johns Hopkins had completed treatment in a cohort of patients with colorectal cancer, with only one response. Not enough, usually, to be a signal. But this patient was special, and it was an exquisite response. The physicians discussing the case were puzzled. A pathologist asked if the patient might have Lynch syndrome. The treating physician noted that he did, and the pathologist remarked that these patients have an unusually dense infiltration of T lymphocytes, a disease hallmark. Therefore, such an individual might be expected to show response. And the rest is history.

This story has several lessons. The first is about team science; the participating pathologist brought information that the average oncologist might not know. That one contribution paved the way for 2 subsequent trials in MSI-H colorectal and noncolorectal tumors. The second is about clinical observation; when you see something extraordinary, ask why. Throughout history, we have learned so much from astute clinicians who took the time to dig deeper and understand better. The third lesson is that the FDA can move quickly. The approval was based on a single-arm trial involving 90 patients with colorectal cancer and 59 patients with 1 of 14 other cancer types. ${ }^{1}$ Patients with end-stage cancer experienced responses, even complete responses, and the responses were durable.

Looking back, it was serendipity that allowed that one patient with Lynch syndrome in the early trial to show us the way. Thank goodness for that! Going forward, the FDA will require more confirmative studies. What that confirmation might look like is a bit mind-boggling, so I'll leave it to the experts. In the meantime, we've got a new option for an uncommon syndrome. And that's a very good thing.

\section{Reference}

1. Le DT, Durham JN, Smith KN, et al. Mismatch-repair deficiency predicts response of solid tumors to PD-1 blockade. Science, doi:10.1126/science.aan6733.

What do you think? Please e-mail correspondence (include contact information) to JNCCN@nccn.org or log into www.editiorialmanager.com/JNCCN to submit a Letter to the Editor.

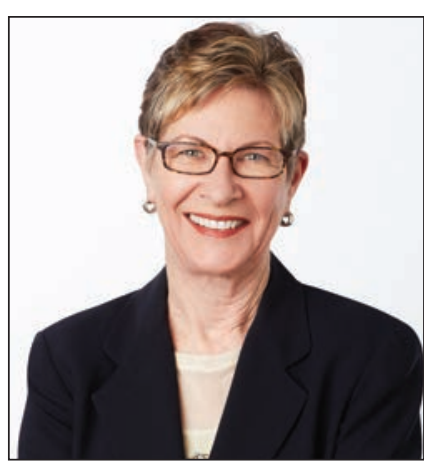

Margaret Tempero, MD

Margaret Tempero, MD, is a Professor of Medicine and Director of the UCSF Pancreas Center and editor-in-chief of JNCCN. Her research career has focused on pancreatic ductal adenocarcinoma, especially in the area of investigational therapeutics. Dr. Tempero has served on the ASCO Board of Directors and as ASCO President. She currently serves on the ASCO Conquer Cancer Foundation Board. She codirected the AACR/ASCO Methods in Clinical Cancer Research and taught this course and similar courses in Europe and Australia. She was founding Chair of the $\mathrm{NCl}$ Clinical Oncology Study Section and served as a member and Chair of the $\mathrm{NCl}$ Board of Scientific Counselors Subcommittee A. She is a member of the Scientific Steering Committee and Chair of the Clinical and Translational Study Section for the Cancer Prevention \& Research Institute of Texas. She is or has been on the Scientific Advisory Boards of the Lustgarten Foundation, the Pancreatic Cancer Action Network, the V Foundation, The Alberta Canada Cancer Board, and the EORTC. She served as a member of the Oncology Drug Advisory Committee for the FDA. She has served as Deputy Director and Interim Director for the UNMC Eppley Cancer Center. She is Chief Emeritus of the Division of Medical Oncology at UCSF and served as the founding Deputy Director and Director of Research Programs at the UCSF Helen Diller Family Comprehensive Cancer Center.

doi:10.6004/jnccn.2017.0141

The ideas and viewpoints expressed in this editorial are those of the author and do not necessarily represent any policy, position, or program of NCCN. 